\title{
Effect of quenched size polydispersity on the fluid-solid transition in charged colloidal suspensions
}

\author{
Jader Colombo ${ }^{1, a)}$ and Marjolein Dijkstra ${ }^{2}$ \\ ${ }^{1}$ Dipartimento di Fisica e Matematica, Università dell'Insubria, Via Valleggio 11, 22100 Como, Italy \\ ${ }^{2}$ Soft Condensed Matter, Debye Institute for NanoMaterials Science, Utrecht University, Princetonplein 5 , \\ 3584 CC, Utrecht, The Netherlands
}

(Received 14 February 2011; accepted 28 March 2011; published online 18 April 2011)

\begin{abstract}
We study the effect of quenched size polydispersity on the phase behavior of charged colloidal suspensions using free-energy calculations in Monte Carlo simulations. The colloids are assumed to interact with a hard-core repulsive Yukawa (screened-Coulomb) interaction with constant surface potential, so that the particles are polydisperse both in size and charge. In addition, we take the size distribution to be fixed in both the fluid and crystal phase (no size fractionation is allowed). We study the fluid-solid transition for various screening lengths and surface potentials, finding that upon increasing the size polydispersity the freezing transition shifts toward higher packing fractions and the density discontinuity between the two coexisting phases diminishes. Our results provide support for a terminal polydispersity above which the freezing transition disappears. () 2011 American Institute of Physics. [doi:10.1063/1.3580284]
\end{abstract}

\section{INTRODUCTION}

The discovery that a system of hard spheres undergoes a first-order freezing transition is undoubtedly one of the milestones of computer simulations applied to statistical mechanics. In the mid-1950s the existence of such a transition was an important open question and no convincing answer could be given starting from first principles. Therefore, it was no surprise that at a symposium held in 1957 at the Stevens Institute of Technology in Hoboken, New Jersey, during a discussion led by G. E. Ulhenbeck a vote on this question taken among prominent scientists, including several Nobel laureates, ended in a draw. ${ }^{1}$ The hesitation of half of the audience is understandable, because the fact that purely repulsive particles can form a stable crystal is far from obvious. The question was finally settled in favor of the existence of a fluid-crystal transition thanks to the seminal molecular dynamics simulations by Alder and Wainwright ${ }^{2}$ and Monte Carlo simulations by Wood and Jacobson, ${ }^{3}$ as well as the later and conclusive Monte Carlo study by Hoover and Ree. ${ }^{4}$ In more recent years, owing to notable advances in the synthesis of colloidal particles, the existence of the phase transition has also been confirmed by extensive experimental evidence: the classic investigation of Pusey and van Megen ${ }^{5}$ reported the spontaneous formation of beautiful hard-sphere crystals.

It is now widely accepted that excluded-volume interactions and repulsive short-range forces play a major role in determining the existence of a freezing transition: indeed, the latter is found in the high-density end of the phase diagram of most systems containing particles with a core. This is the case of hard-core repulsive Yukawa (or screened Coulomb) fluids, which have been extensively adopted as models for suspensions of charge-stabilized colloids ${ }^{6}$ proving quite successful

\footnotetext{
a) Author to whom correspondence should be addressed. Electronic mail: jader@ifb.baug.ethz.ch. Present address: Institut für Baustoffe (IfB) ETH Zürich, HIF E 16 Schafmattstr. 6, 8093 Zürich, Switzerland.
}

in their use as long as the standard DLVO theory ${ }^{7}$ is applicable and the Van der Waals interactions are negligible. One of the main features in the phase diagram of such systems is a freezing transition between a fluid and a crystalline solideither a body-centered cubic (bcc) or a face-centered cubic (fcc) phase depending on the inverse Debye screening length and the volume fraction-which has been consistently observed in experiments ${ }^{8}$ and predicted by theory ${ }^{9,10}$ and simulation. ${ }^{11}$

A feature of colloidal dispersions that is not considered in the simple hard-core Yukawa model is polydispersity, i.e., a stochastic variation in one or more properties (for instance size, shape, or charge) of the particles. That polydispersity might have a deep impact on the freezing transition is intuitive, since the very idea of a periodic, regular lattice is somewhat at odds with a random variation in any property of the particles. Indeed, polydisperse systems usually have different—and richer-phase diagrams if compared to their monodisperse counterparts. ${ }^{12}$ While a small degree of polydispersity is expected to introduce a mere distortion in the phase boundaries, leaving the topology of the phase diagram largely unaffected, higher degrees of polydispersity might significantly change the phase behavior of the system giving birth to new phases or suppressing existing phase transitions. In this regard, a clear distinction should be made between the general case and the case we will address, namely, the one of quenched polydispersity.

In order to elucidate this point, let us focus on the freezing transition of a fluid whose particles are polydisperse in size. The composition of the whole system, regardless of its phase behavior, is quantified by the so-called parent size distribution, which determines the probability that any given particle has a certain diameter. If the system separates into a fluid and a solid, then a daughter size distribution is associated with each phase. Attainment of thermodynamic equilibrium generally implies that the particles fractionate (i.e., distribute 
themselves unevenly) over the two phases, so that the daughter size distributions differ from the parent. Fractionation is indeed the key element bestowing richness and variety to polydisperse phase diagrams. However, in real experiments size fractionation might suffer from dynamical slowing down, ${ }^{13-16}$ or even be suppressed on purpose by compressing the system sufficiently fast to high density by centrifugal forces, gravity, or dielectric compression. ${ }^{17,18}$ If fractionation is completely avoided the daughter size distributions are necessarily equal to the one of the parent phase: we refer to this scenario with the term quenched polydispersity.

The phase behavior of polydisperse hard spheres, the cleanest polydisperse system in which the freezing transition may be studied, has been the subject of extensive investigation in the literature. For instance, it is well-known that hard spheres with a sufficient amount of size polydispersity can form upon compression glassy states instead of crystalline solids. ${ }^{13-16,19-22}$ The question concerning the existence of a terminal polydispersity above which no stable crystal can exist has prompted a substantial amount of theoretical ${ }^{23-29}$ and computational ${ }^{16,30-35}$ work devoted to the determination of an accurate equilibrium phase diagram for polydisperse hard spheres.

Conversely, to the best of our knowledge no similar effort has been devoted to polydisperse hard-core Yukawa fluids. Therefore, in this work we plan to (i) elucidate the effect of polydispersity on the liquid-solid phase boundary, and its dependence on the parameters of the Yukawa tail; (ii) ascertain the existence of a terminal polydispersity above which the crystal phase is suppressed in favor of a glassylike state; (iii) check whether the phenomenon of re-entrant melting predicted for hard spheres ${ }^{25}$ takes place. We tackle the problem by performing free energy calculations via Monte Carlo simulation, which allows us to map out the phase boundaries. We shall restrict ourselves to the case of quenched polydispersity, which is easier to deal with but also has practical relevance for experiments that exploit size polydispersity to make amorphous and glassy states.

\section{MODEL}

\section{A. Interaction energy}

We consider a system of $N$ spherical particles, polydisperse in size, enclosed in a box of volume $V$. Let $\boldsymbol{r}_{i}$ denote the spatial coordinate of particle $i$, and let $\sigma_{i}$ and $a_{i}=\sigma_{i} / 2$ be its diameter and radius, respectively. The pair potential $v\left(r_{i j}\right)$ is taken as pairwise additive, comprising a hard-core and a repulsive Yukawa tail:

$$
v\left(r_{i j}\right)= \begin{cases}+\infty & r_{i j}<a_{i}+a_{j} \\ \epsilon_{i j} \frac{a_{i}+a_{j}}{r_{i j}} e^{-\kappa\left(r_{i j}-a_{i}-a_{j}\right)} & \text { otherwise }\end{cases}
$$

where $r_{i j}=\left|\boldsymbol{r}_{i}-\boldsymbol{r}_{j}\right|$ and $\kappa$ is the inverse screening length.

The contact value $\epsilon_{i j}$ of the pair potential in the context of the standard DLVO theory is given by Ref. 7

$$
\epsilon_{i j}=\frac{\beta^{-1} Z_{i} Z_{j}}{\left(1+\kappa a_{i}\right)\left(1+\kappa a_{j}\right)} \frac{\lambda_{B}}{a_{i}+a_{j}},
$$

where $\beta=1 / k_{B} T, k_{B}$ is the Boltzmann constant and $T$ the temperature, $Z_{i}$ and $Z_{j}$ are the charge number of particle $i$ and $j$, respectively, expressed in units of the elementary charge and $\lambda_{B}=e^{2} / \varepsilon k_{B} T$ is the Bjerrum length with $e$ the elementary charge and $\varepsilon$ the dielectric constant of the solvent. In practice the charge of a particle will be a (possibly stochastic) function of its size, so a system polydisperse in size will also be polydisperse in charge: the simplest case is that of constant surface charge density, according to which the particle charge is proportional to the square of its linear size. Another important condition, which is often met in colloidal dispersions and therefore will be adopted in this work, is the one of constant surface potential, i.e., the electrostatic potential measured on the surface of a particle is the same for each particle in the system, regardless of its radius. In the context of linear Poisson-Boltzmann theory, which underlies the derivation of the screened Coulomb part in the standard DLVO potential (1), the electrostatic potential $\Psi_{i}$ on the surface of a single, isolated particle $i$ embedded in the electrolyte reads as follows: ${ }^{7}$

$$
\Psi_{i}=(\beta e)^{-1} \frac{Z_{i}}{1+\kappa a_{i}} \frac{\lambda_{B}}{a_{i}} .
$$

If the electrostatic potential is taken as a constant $\left(\Psi_{i}=\Psi\right)$, independent of particle number $i$, Eqs. (2) and (3) imply

$$
\epsilon_{i j} \propto \frac{a_{i} a_{j}}{a_{i}+a_{j}},
$$

the constant of proportionality being the same for all pairs. If, moreover, we choose a reference particle diameter $\bar{\sigma}$ and call $\bar{\epsilon}$ the contact value for the pair interaction of two particles with reference size $\bar{\sigma}$, we can express the pair interaction of any two particles in the system in the following way:

$$
v\left(r_{i j}\right)= \begin{cases}+\infty & r_{i j}<a_{i}+a_{j} \\ \bar{\epsilon} \frac{\sigma_{i} \sigma_{j}}{\bar{\sigma} r_{i j}} e^{-\kappa\left(r_{i j}-a_{i}-a_{j}\right)} & \text { otherwise }\end{cases}
$$

This will be taken as the final expression defining the potential energy of the system, being conveniently characterized by the two parameters $\bar{\epsilon}$ and $\kappa$, besides the typical particle diameter $\bar{\sigma}$.

In order to make contact with experiments, one can also show from Eqs. (2) and (3) that

$$
\beta \bar{\epsilon}=(\beta e \Psi)^{2} \frac{\bar{\sigma}}{4 \lambda_{B}} ;
$$

typical values for a colloid in a low dielectric solvent at room temperature $\operatorname{are}^{8} \Psi=25 \mathrm{mV}, \lambda_{B} / \bar{\sigma}=0.01$, which roughly corresponds to $\beta \bar{\epsilon} \approx 20$.

\section{B. Polydispersity}

The polydispersity of the system is most easily modeled by a normalized size distribution $\mathcal{N}(\sigma)$, which is defined such that the number of particles with a diameter between $\sigma$ and $\sigma+d \sigma$ is given by $N \mathcal{N}(\sigma) d \sigma$. In the absence of a clear experimental indication, the choice of $\mathcal{N}(\sigma)$ is somewhat arbitrary. The size polydispersity $p$ is defined as the ratio between the standard deviation and the mean value of the size 
distribution:

$$
p=\frac{\sqrt{\overline{\sigma^{2}}-\bar{\sigma}^{2}}}{\bar{\sigma}},
$$

where the averages $\bar{\sigma}$ and $\overline{\sigma^{2}}$ are defined by

$$
\bar{\sigma}=\int d \sigma \sigma \mathcal{N}(\sigma), \quad \overline{\sigma^{2}}=\int d \sigma \sigma^{2} \mathcal{N}(\sigma) .
$$

In this work, we assume that the particle diameters follow a log-normal distribution:

$$
\mathcal{N}(\sigma)=\frac{1}{z \sqrt{2 \pi} \sigma} \exp \left[-\frac{\left(\log (\sigma / \bar{\sigma})+z^{2} / 2\right)^{2}}{2 z^{2}}\right],
$$

with $z=\sqrt{\log \left(1+p^{2}\right)}$. No particular reason supports this choice; viable alternatives, also found in literature include top-hat, triangular, and Gamma (Schultz) distributions. However, once the polydispersity index $p$ is fixed we expect the results to depend not too markedly on the shape of the distribution.

Equation (9) defines the parent distribution: the quantity $N \mathcal{N}(\sigma) d \sigma$ corresponds to the total number of particles in the box having a diameter between $\sigma$ and $\sigma+d \sigma$. If the system phase separates into two phases, then a daughter distribution function $\mathcal{D}_{\omega}(\sigma)$ can be attributed to each phase $\omega(\omega=\mathrm{I}, \mathrm{II})$ in such a manner that $N_{\omega} \mathcal{D}_{\omega}(\sigma) d \sigma$ is the number of particles in phase $\omega$ with a diameter lying in the interval $[\sigma, \sigma+d \sigma]$, $N_{\omega}$ being the total number of particles belonging to that phase. Conservation of particle numbers requires that

$$
\begin{gathered}
N_{\mathrm{I}}+N_{\mathrm{II}}=N, \\
N_{\mathrm{I}} \mathcal{D}_{\mathrm{I}}(\sigma)+N_{\mathrm{II}} \mathcal{D}_{\mathrm{II}}(\sigma)=N \mathcal{N}(\sigma) .
\end{gathered}
$$

The constraint of quenched polydispersity forces the daughter size distributions to match that of the parent phase:

$$
\mathcal{D}_{\mathrm{I}}(\sigma)=\mathcal{D}_{\mathrm{II}}(\sigma)=\mathcal{N}(\sigma) .
$$

If the system is allowed to fractionate, i.e., particles with diameter $\sigma$ may distribute themselves unevenly over the two daughter phases, not a priori constraint exists, as long as Eq. (10) is satisfied together with the conditions for thermodynamic equilibrium, as will be explained below.

\section{Reminder on polydisperse phase equilibria}

A polydisperse system can be considered as a multicomponent mixture with an infinite number of species. However, several subtleties arise and care must be taken to properly define thermodynamic quantities. In the polydisperse limit, the thermodynamic potential of a homogeneous bulk phase depends on the usual thermodynamic variables and becomes a functional of the size distribution function. For instance, the Helmholtz free energy $F$ of a homogeneous daughter phase with a size distribution $\mathcal{D}(\sigma)$ is given by ${ }^{36}$

$$
F=F(T, V ; N \mathcal{D}(\sigma)) .
$$

Subsequently, one can obtain the pressure by $P(T, \rho$; $\mathcal{D}(\sigma))=(-\partial F / \partial V)_{N \mathcal{D}(\sigma), T}$, where $\rho=N / V$ denotes the particle number density, whereas the chemical potential $\mu$ (which is now a function of the particle diameter $\sigma$ ) is defined through a functional derivative:

$$
\mu(\sigma, T, \rho ; \mathcal{D}(\sigma))=\left(\frac{\delta F}{\delta N \mathcal{D}(\sigma)}\right)_{V, T} .
$$

The conditions for phase coexistence are given by a straightforward generalization of those for a mixture with a finite number of components: ${ }^{36}$

$$
\begin{aligned}
T_{\mathrm{I}} & =T_{\mathrm{II}}, \\
P_{\mathrm{I}}\left(T_{\mathrm{I}}, \rho_{\mathrm{I}} ; \mathcal{D}_{\mathrm{I}}(\sigma)\right) & =P_{\mathrm{II}}\left(T_{\mathrm{II}}, \rho_{\mathrm{II}} ; \mathcal{D}_{\mathrm{II}}(\sigma)\right), \\
\mu_{\mathrm{I}}\left(\sigma, T_{\mathrm{I}}, \rho_{\mathrm{I}} ; \mathcal{D}_{\mathrm{I}}(\sigma)\right) & =\mu_{\mathrm{II}}\left(\sigma, T_{\mathrm{II}}, \rho_{\mathrm{II}} ; \mathcal{D}_{\mathrm{II}}(\sigma)\right) \quad \forall \sigma .
\end{aligned}
$$

Equation (14), when supplemented with Eq. (10), forms the thermodynamic basis for studying phase equilibria in polydisperse systems.

A substantial simplification arises if the constraint (11) of quenched polydispersity is imposed. In that case, the functional dependence on the size distribution function $\mathcal{D}(\sigma)$ becomes immaterial and the Helmholtz free energy $F(T, V, N)$ is simply a function of temperature, volume, and number of particles. As a consequence, the dependence on $\mathcal{D}(\sigma)$ drops out for the pressure $P(T, \rho)$ and the chemical potential reduces to that for a one-component system:

$$
\mu(T, \rho)=\left(\frac{\partial F}{\partial N}\right)_{V, T, \mathcal{D}(\sigma)}=\int \mu(\sigma, T, \rho ; \mathcal{D}(\sigma)) \mathcal{D}(\sigma) d \sigma
$$

Moreover, the conditions (14) for phase coexistence can be cast in the same form as the ones for a monodisperse system:

$$
\begin{aligned}
T_{\mathrm{I}} & =T_{\mathrm{II}}, \\
P_{\mathrm{I}}\left(T_{\mathrm{I}}, \rho_{\mathrm{I}}\right) & =P_{\mathrm{II}}\left(T_{\mathrm{II}}, \rho_{\mathrm{II}}\right), \\
\mu_{\mathrm{I}}\left(T_{\mathrm{I}}, \rho_{\mathrm{I}}\right) & =\mu_{\mathrm{II}}\left(T_{\mathrm{II}}, \rho_{\mathrm{II}}\right) .
\end{aligned}
$$

Owing to these simplifications, once the Helmholtz free energy is known for both phases a standard common tangent construction $^{37}$ can be used to locate the densities of the coexisting phases.

\section{METHODS}

The task is now to devise a Monte Carlo strategy to determine, at fixed temperature, the densities of the coexisting fluid (F) and crystalline solid (S) phases in a hard-core repulsive Yukawa system in the quenched polydispersity case. Concerning the crystalline solid phase, the most natural choice for the lattice structure is the one being stable in the monodisperse limit, but other choices can be made as well.

The size polydispersity of the system is described by one of the realizations of the entire stochastic set $\left\{\Sigma_{i}\right\}$, that is one of the possible assignments of sizes to the particles drawn in accordance with the underlying probability distribution function. As a first remark, we note that the definition for the Helmholtz free energy $F(T, V, N)$ for quenched polydispersity is stochastic: formally, the free energy of a polydisperse finite system is a function of the entire stochastic set $\left\{\Sigma_{i}\right\}$ of particle diameters: only in the thermodynamic limit it becomes a true functional of the size distribution function $\mathcal{N}(\sigma) .^{52}$ To deal with the finite size of the system 
inherent to a Monte Carlo simulation, at least two strategies are viable: either we explore different realizations of the polydisperse system in the course of the same simulation (for example devising moves which change the size of the particles), or we perform a separate simulation for a number of realizations and then average the results a posteriori. In this work, we pursued the second approach. In order to ensure a good sampling of the disorder, the size of the simulated system and the number of independent realizations should be balanced.

\section{A. Fluid and solid free energy}

Free energy calculations via Monte Carlo simulation are notoriously a time-demanding task, so the number of direct free energy evaluations should be kept to a minimum. At fixed temperature once the free energy of a homogeneous phase is known at a reference density $\rho^{0}$, the entire branch of the free energy can be determined provided the equation of state for the same phase (in the form of the pressure as a function of density) is available. This is most readily seen by defining the dimensionless free energy per particle

and hence,

$$
f(T, \rho)=\frac{\beta F}{N},
$$

$$
f(T, \rho)=f\left(T, \rho^{0}\right)+\int_{\rho^{0}}^{\rho} \frac{\beta P(T, \varrho)}{\varrho^{2}} d \varrho .
$$

The equations of state for both the fluid and the crystalline solid phase can be computed using standard computer simulations in the $N P T$ ensemble ${ }^{39}$ the remaining task is thus to estimate the reference part $f\left(T, \rho^{0}\right)$.

To this end, we first split the free energy in the sum of an ideal gas term and an excess term:

$$
f(T, \rho)=f^{\mathrm{id}}(T, \rho)+f^{\mathrm{ex}}(T, \rho) .
$$

The ideal gas term for a polydisperse system with a generic size distribution function $\mathcal{D}(\sigma)$ can be derived (with some caveats involving logarithmically divergent quantities) from the equivalent expression for a multicomponent mixture; the final result reads as follows: ${ }^{36}$

$$
f^{\mathrm{id}}(T, \rho ; \mathcal{D}(\sigma))=\int d \sigma \mathcal{D}(\sigma)\left[\log \left(\rho \mathcal{D}(\sigma) \Lambda^{3}(\sigma)\right)-1\right],
$$

where $\Lambda(\sigma)$ stands for the thermal de Broglie wavelength, which has a dependence on the size of the particle through its mass. Defining an effective thermal wavelength $\bar{\Lambda}$ (or equivalently an effective mass $\bar{m}$ ) so that

$$
\log \bar{\Lambda}^{3}=\int d \sigma \mathcal{D}(\sigma) \log \left(\Lambda^{3}(\sigma)\right),
$$

Equation (19) can be recast in the suggestive form

$$
f^{\mathrm{id}}(T, \rho ; \mathcal{D}(\sigma))=\log \left(\rho \bar{\Lambda}^{3}\right)-1+\int d \sigma \mathcal{D}(\sigma) \log (\mathcal{D}(\sigma)),
$$

that is, the ideal gas free energy density of a polydisperse system equals that of a monodisperse, one-component system comprising particles with an effective mass $\bar{m}$ plus an ideal mixing term involving the distribution function $\mathcal{D}(\sigma)$. Under the constraint of quenched polydispersity, the size distribution function of the two daughter phases equals that of the parent distribution $\mathcal{D}_{I}(\sigma)=\mathcal{D}_{I I}(\sigma)=\mathcal{N}(\sigma)$, and hence $f^{\text {id }}(T, \rho ; \mathcal{D}(\sigma))=f^{\text {id }}(T, \rho ; \mathcal{N}(\sigma))$. In this particular case, the contribution arising from the entropy of mixing is identical in both daughter phases and can be neglected in the determination of phase equilibria. The same holds for the additive constant involving the effective thermal wavelength $\bar{\Lambda}$, therefore particle masses play no role either.

In the fluid phase, the dilute limit where the excess contribution vanishes can be reached starting from any density without incurring a phase transition, so the entire branch of the free energy density of the fluid phase $f_{\mathrm{F}}(T, \rho)$ can be computed from the knowledge of the equation of state alone:

$$
f_{\mathrm{F}}(T, \rho)=f_{\mathrm{F}}^{\mathrm{id}}(T, \rho)+\int_{0}^{\rho} \frac{\beta P_{\mathrm{F}}(T, \varrho)-\varrho}{\varrho^{2}} d \varrho
$$

The same does not hold in the crystalline solid phase, and the need arises for a direct evaluation of the reference free energy density $f_{\mathrm{s}}\left(T, \rho_{\mathrm{S}}^{0}\right)$. To fulfill this goal a number of approaches exist; in this work we adopted the method of Frenkel and Ladd (also termed Einstein integration), which appears to be the most widely used and tested to date. In the following we will just introduce its main features referring the interested reader to the original articles ${ }^{40,41}$ for further details.

The key idea of the method is to build a reversible thermodynamic path between the system of interest and the noninteracting Einstein crystal, i.e., a system of noninteracting particles linked to their respective lattice sites by harmonic springs, whose free energy can be computed exactly. For a system comprising particles with a hard core, this is achieved by introducing an auxiliary potential energy function $U_{\lambda}$ containing a coupling parameter $\lambda$ whose purpose is to smoothly switch off interparticle interactions other than the cores while switching on the lattice springs:

$$
U_{\lambda}\left(\mathbf{r}^{N}\right)=U_{\mathrm{HS}}\left(\mathbf{r}^{N}\right)+(1-\lambda) U\left(\mathbf{r}^{N}\right)+k_{B} T \lambda \alpha \sum_{i=1}^{N} \frac{\left|\mathbf{r}_{i}-\mathbf{R}_{i}\right|^{2}}{\bar{\sigma}^{2}} .
$$

In the previous expression $U_{\mathrm{HS}}\left(\mathbf{r}^{N}\right)$ is the potential energy due to the pairwise sum of hard-sphere potentials, $U\left(\mathbf{r}^{N}\right)$ the remaining part of the interparticle potential, $\alpha$ a dimensionless spring constant common to all particles and $\left\{\boldsymbol{R}_{i}\right\}$ the set of ideal lattice points of the crystal; when $\lambda=0$ the system of interest is recovered, while in the limit $\lambda \rightarrow 1$, provided the spring constant $\alpha$ is chosen sufficiently high to render the presence of the cores immaterial, the noninteracting Einstein crystal is approached. It then follows that the free energy density of the system is given by ${ }^{41}$

$$
\begin{aligned}
f_{\mathrm{S}}(T, \rho)= & \frac{1}{N} \int_{0}^{1} d \lambda\left\langle\beta U\left(\mathbf{r}^{N}\right)-\alpha \sum_{i=1}^{N} \frac{\left|\boldsymbol{r}_{i}-\boldsymbol{R}_{i}\right|^{2}}{\bar{\sigma}^{2}}\right\rangle_{\lambda}^{\mathrm{CM}} \\
& +\frac{3(N-1)}{2 N} \log \left(\frac{\alpha \bar{\Lambda}^{2}}{\pi \bar{\sigma}^{2}}\right)+\frac{1}{N} \log \left(\frac{\bar{\Lambda}^{3}}{V(2 \pi N)^{1 / 2}}\right),
\end{aligned}
$$


where the notation $\langle\cdot\rangle_{\lambda}^{\mathrm{CM}}$ stands for an ensemble average in the $N V T$ ensemble for a system interacting with the potential energy function $U_{\lambda}\left(\mathbf{r}^{N}\right)$ and subject to the constraint of fixed center of mass..$^{53}$

The free energy computed according to Eq. (23) is subject to finite-size effects decaying as $N^{-1}$ : to properly deal with this issue, one usually repeats the calculation varying the number of particles and then extrapolates the result to the thermodynamic limit. ${ }^{40,41}$ In this work we instead stick with a single calculation relying on a large system size, already required to properly account for particle polydispersity, to reduce the spurious effects.

\section{B. Algorithm}

Below we describe in more detail the procedure that we used to map out the phase boundaries. First, we set the parameters of the pair potential (5), choosing the dimensionless reference contact value $\beta \bar{\epsilon}$ and the inverse screening length $\kappa \bar{\sigma}$, and the total number of particles $N$. Moreover, we choose a lattice structure for the polydisperse crystal-typically, we select the structure of the solid coexisting with the fluid in the monodisperse case, the interparticle potential being equaland a density $\rho_{\mathrm{s}}^{0}$ for the reference free energy evaluation. Then, for each value of the polydispersity index $p, n_{r}$ realizations of the system are constructed by drawing random diameters from the log-normal distribution (9). The particles are initially arranged with a density $\rho_{\mathrm{s}}^{0}$ on a regular lattice having the preselected structure: this is achieved by trial and error, positioning the particles one after another at the sites of the lattice as the random diameters are being drawn; as soon as an overlap occurs, the whole procedure is restarted from the very beginning, so that the parent distribution $\mathcal{N}(\sigma)$ is not biased. The procedure becomes inefficient as $p$ and $\rho_{\mathrm{s}}^{0}$ increase and beyond a certain limit the polydisperse crystal cannot be constructed within a reasonable time; a fictitious line (not well-defined as it depends on the number of particles and on the time that one is prepared to wait) can be drawn in the phase diagram in the packing fraction $\eta$ - polydispersity $p$ plane where the polydisperse crystal cannot be constructed and is therefore likely unstable.

Einstein integration is performed on the initial configuration to get the solid free energy at the reference density. We use the same configuration to start the computation of the solid branch of the equation of state, and, after melting the crystal, of the fluid branch. Once all realizations of the polydisperse system have been processed, we take averages over the different outcomes. ${ }^{54}$ Finally, we evaluate the fluid and solid free energy as a function of density by means of Eqs. (22) and (17), respectively, and determine the density of the coexisting phases by imposing the conditions of twophase equilibrium (15). The conversion between density and packing fraction is performed at the end of the whole procedure by using the actual mean volume of the particles computed over the different realizations.

\section{Technical details}

All the simulations are carried out in a cubic box with periodic boundary conditions and the minimum image con- vention; the number of particles is chosen so that in the solid phase an integer number of primitive lattice cells fits into the box. A spherical cutoff is applied to the pair potential (5); the cutoff radius $r_{c}$ is set beforehand and kept constant during the course of a simulation: care is taken to ensure that it never exceeds half of the box length. No tail correction is applied to compensate for the cutoff: on the one hand, the exponential decay of the potential makes the correction negligible if $r_{c}$ is sufficiently large ${ }^{55}$ on the other hand, the actual evaluation of the tail correction is not straightforward in the case of polydispersity.

Equations of state are computed by means of simulations in the NPT ensemble. For a single branch several tens of points are sampled and later interpolated by the virial expansion

$$
\frac{\beta P(T, \rho)-\rho}{\rho^{2}}=\sum_{j=0}^{M} b_{j} \rho^{j},
$$

with fitting coefficients $\left\{b_{j}\right\}$ and a polynomial degree $M \lesssim 10$.

To compute the reference solid free energy within the Frenkel-Ladd method, the integral in Eq. (23) is evaluated by a numerical quadrature rule using a total of thirty nodes. We divide the interval spanned by $\lambda$ in the two subintervals $[0-0.2)$ and $[0.2,1.0]$, and apply to each one the $15-7$ Gauss-Kronrod rule, ${ }^{42,43}$ which has the advantage over the commonly used Legendre rule of providing an estimate of the error in the result. The rationale behind the splitting is that the integrand is steeper near the left end of the interval, where the springs are less effective in confining the particles close to the lattice sites, therefore, it is convenient to concentrate the quadrature nodes in that region. We set the value of the spring constant $\alpha$ sufficiently high so that the noninteracting regime is reached when $\lambda=1$. We verify this by comparing the average value of the internal energy with the value expected for the harmonic potential of the springs on the basis of the equipartition theorem. ${ }^{56}$

The conditions of two-phase equilibrium (15) are handled by standard solvers of nonlinear equations: the chemical potential $\mu$ is computed without performing derivatives by means of the thermodynamic identity

$$
\beta \mu(T, \rho)=f(T, \rho)+\frac{\beta P(T, \rho)}{\rho} .
$$

Simulations are accelerated by a combination of high and low level optimizations. A cell-list method is used to track the position of the particles: a regular grid of cells, able to expand and shrink along with the box, is set up so that on average a cell contains only a few particles. The distance between each pair of cells - expressed in units of the box length, in order to be unaffected by scaling operations-is precomputed at the beginning of the simulation, and a list is associated with each cell with pointers to the other cells sorted in order of increasing distance: in this way, the set of cells that are within the cutoff distance from any given cell can be identified at constant computational cost without performing any additional distance calculation. Moreover, the single-instruction, multiple-data extensions of Intel and AMD processors are 
TABLE I. Summary of the parameters characterizing the four different model systems that have been simulated. From left to right: label of the model; dimensionless contact value of the pair potential $\beta \bar{\epsilon}$, inverse screening length $\kappa \bar{\sigma}$ and cutoff radius $r_{c} / \bar{\sigma}$ of the pair potential; lattice structure (FCC or BCC) and packing fraction $\eta_{\mathrm{S}}^{0}$ coexisting with the fluid in the monodisperse case (for systems A-C the estimate is based on the phase diagrams found in Ref. 11; for system D the mapping of the hard-core Yukawa model onto the point Yukawa model devised in the same article has been applied); number of particles $N$; number of realizations $n_{r}$ per polydispersity value over which averages were taken; dimensionless spring constant $\beta \alpha \bar{\sigma}^{2}$ of the Einstein crystal; maximum value of the polydispersity $p_{\max }$ for which fluid-solid coexistence could be determined.

\begin{tabular}{rrrrllllll}
\hline \hline Lbl & $\beta \bar{\epsilon}$ & $\kappa \bar{\sigma}$ & $r_{c} / \bar{\sigma}$ & Lattice & $\eta_{\mathrm{S}}^{0}$ & $N$ & $n_{r}$ & $\beta \alpha \bar{\sigma}^{2}$ & $p_{\max }$ \\
\hline A & 20 & 10 & 3.0 & FCC & 0.29 & 5324 & 5 & 4000 & 0.095 \\
B & 81 & 3.333 & 5.0 & BCC & 0.108 & 4394 & 5 & 4000 & 0.125 \\
C & 20 & 3.333 & 4.0 & BCC & 0.28 & 4394 & 5 & 4000 & 0.100 \\
D & 1200 & 0.7 & 20.0 & BCC & 0.0029 & 4394 & 5 & 100 & 0.220 \\
\hline \hline
\end{tabular}

exploited to achieve a limited amount of low-level parallelism in the computation of particle interactions.

\section{RESULTS AND DISCUSSION}

We now apply the procedure outlined in Sec. III to a number of hard-core Yukawa systems. Four different systems, which we label with letters from A to D, are considered: a detailed list of the parameters defining each system can be found in Table I. We only take into account the solid phase that coexists with the fluid phase in the absence of polydispersity, all other parameters for the screening length and reference contact value being equal. It is indeed possible that beyond a certain degree of polydispersity another structure might be stable, but this issue has not been addressed here.

Systems A,B,C are models of micrometer-sized colloids with a surface potential in the range $25-50 \mathrm{mV}$ dispersed in a low dielectric solvent at room temperature; the parameters were chosen to allow a comparison with data available in the literature for the monodisperse case. ${ }^{11}$

System D is devised to mimic the experiments involving highly charged water-in-oil droplets reported by Leunissen et al. ${ }^{44}$ in which a stable crystalline solid at extremely low packing fraction and high polydispersity was seen. No details are to be found in the original article, but a rough analysis of the pictures reveals a packing fraction in the order of $10^{-3}$ and a polydispersity as high as $20 \%$. The parameters of the potential are set according to the mapping of the hard-core Yukawa model onto the point Yukawa model devised in Ref. 11 in such a way that in the absence of polydispersity the fluid-solid transition occurs at a packing fraction of the same order of the aforementioned estimate.

\section{A. Phase diagrams}

The phase behavior of system $\mathrm{A}$ is presented in the packing fraction, polydispersity $(\eta, p)$ plane in Fig. 1. In the absence of polydispersity the phase boundaries are fully consistent with the results of Hynninen and Dijkstra ${ }^{11}$ (see also the column $\eta_{\mathrm{s}}^{0}$ of Table I). As the polydispersity increases, two phenomena occur: (i) the freezing transition shifts toward higher packing fractions; (ii) the difference $\Delta \eta$ between the packing fraction of the solid and the packing fraction of the fluid decreases. This trend, made explicit in the inset of the figure, suggests the existence of a terminal polydispersity $p_{t}$ at which the density gap closes $(\Delta \eta=0)$. The main diagram also shows that just below $p_{t}$ re-entrant melting takes place, that is the solid melts upon compression.

To gain a better understanding of these features, in Fig. 2 the difference between the chemical potential of the coexisting solid and fluid phase $\Delta \mu$ is plotted as a function of the pressure $\beta P \bar{\sigma}^{3}$ for several values of the polydispersity $p$ : in such a diagram, phase coexistence corresponds to $\Delta \mu=0$, whereas $\Delta \mu \lessgtr 0$ marks the stability of a single phase. For most values of the polydispersity the curve shows only one intersection with the $\Delta \mu=0$ axis, which marks the fluid-toordered solid transition. For sufficiently high polydispersity, however, a second intersection appears, and a disordered, fluidlike configuration becomes favoured again when the pressure is raised above a prescribed value. The terminal polydispersity $p_{t}$ may be defined as the one corresponding to a curve tangent to the horizontal axis. Finally, when $p>p_{t}$ the curve lies entirely in the region of stability of the single fluid

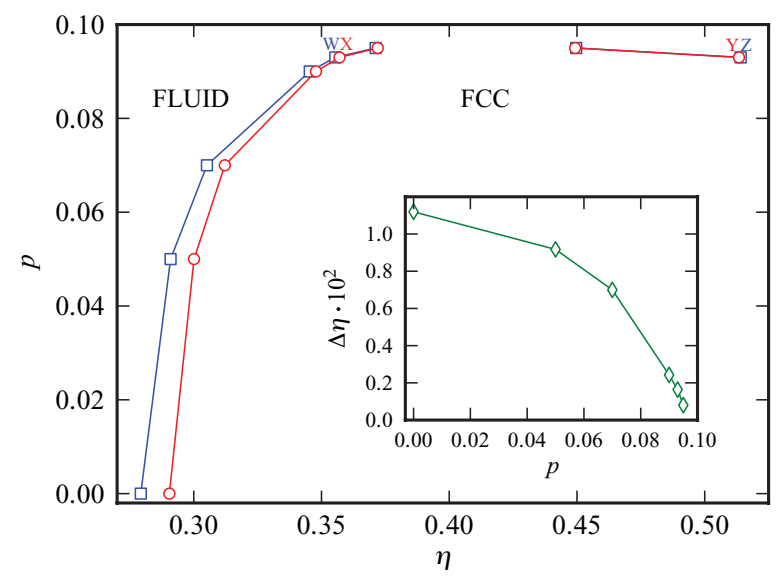

FIG. 1. Quenched-polydispersity phase diagram of hard-core Yukawa particles with $\beta \bar{\epsilon}=20, \kappa \bar{\sigma}=10$ (system A) presented in the packing fraction $\eta$ - polydispersity $p$ plane. Symbols denote the results from Monte Carlo simulations, whereas lines serve as guides to the eye. Re-entrant melting can be seen in the upper right part of the diagram, corresponding to polydispersity $p=0.093$ and $p=0.095$. "FCC" denotes the stable face-centered-cubic phase and "FLUID" the stable fluid phase. The four state points denoted by $\mathrm{W}-\mathrm{Z}$ are object of structural analysis later in the text. In the inset, the difference between the packing fraction of the solid and the one of the fluid across the main (that is, nonre-entrant) freezing transition is plotted as a function of the polydispersity $p$. 


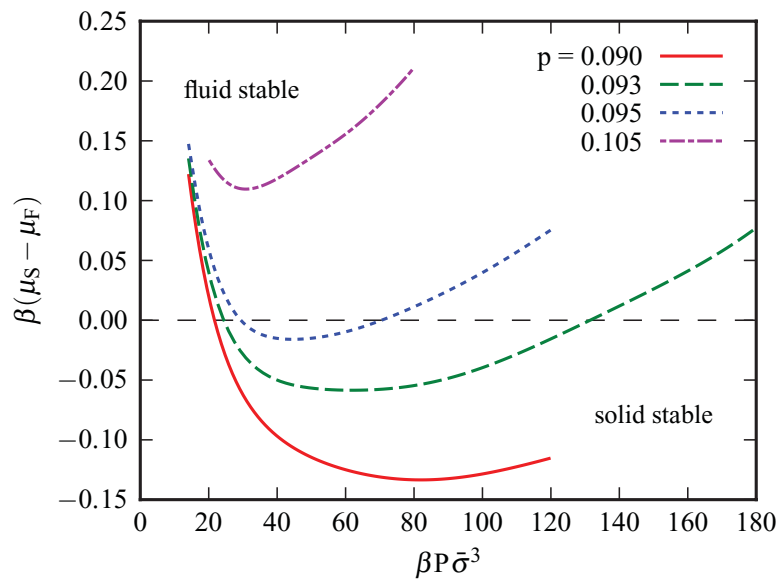

FIG. 2. System A: difference between the chemical potential of the solid $\mu_{\mathrm{S}}$ and the chemical potential of the fluid $\mu_{\mathrm{F}}$ plotted as a function of the pressure for a choice of indices of polydispersity.

phase and both the freezing transition and the re-entrant melting transition disappear. ${ }^{57}$

This scenario closely matches the one first predicted by Bartlett and Warren ${ }^{25}$ and later confirmed by simulations by Nogawa et al. ${ }^{16}$ for a hard-sphere system. Although close to the terminal polydispersity the results might be affected by numerical uncertainties inherent to any thermodynamic integration and to a lesser extent to equation of state calculations, we believe that the topology of the phase diagram as depicted in Fig. 1 is sound. The precise identification of the terminal polydispersity $p_{t}$, besides being prone to the aforementioned numerical errors, would cost a great computational effort. Nonetheless, a rough estimate is readily provided: by extrapolating the curve depicted in the inset of Fig. 1 the terminal polydispersity can be bracketed as $0.095<p_{t}<0.100 .{ }^{58}$

As a reference, we also show in Table II the variation of the solid free energy between different realizations of the polydispersity. For each value of polydispersity $p$, and for each realization of the polydispersity we have generated, we report the excess part of the dimensionless free energy per particle $f_{\mathrm{s}}$ computed at the reference density $\rho_{\mathrm{s}}^{0}$ by means of the Frenkel-Ladd method. The different realizations are labeled with the first four moments of the sample diameter distribution: mean $(m)$, standard deviation $(s)$, skewness $\left(\gamma_{1}\right)$, and excess kurtosis $\left(\gamma_{2}\right)$. The excess free energy in the absence of polydispersity is also shown.

We now discuss model system B, characterized by stronger and more long-ranged interparticle interactions. The phase behavior is shown in Fig. 3 in the packing fraction $\eta$ - polydispersity $p$ plane. Due to the stronger repulsion, freezing takes place at packing fractions significantly lower than the ones typical of the previous system; apart from that, the phase behavior is entirely similar. We stress two minor differences: (i) re-entrant melting could only be determined for the highest value of the polydispersity $p=0.125$ just before the disappearance of the freezing transition at $p \simeq 0.130$ (see the caption of the figure); (ii) the solid supports a higher degree of polydispersity than what seems possible in system A: indeed, the terminal polydispersity can be estimated as $0.125<p_{t}<0.130$.
TABLE II. System A: variation of the excess part of the dimensionless solid free energy $f_{\mathrm{S}}^{\mathrm{ex}}=\beta F_{\mathrm{S}}^{\mathrm{ex}} / N$ at the reference density $\rho_{\mathrm{S}}^{0}$ between different realizations of the polydispersity, which are characterized by the first four moments of the diameter distribution: mean $(m)$, standard deviation $(s)$, skewness $\left(\gamma_{1}\right)$, and excess curtosis $\left(\gamma_{2}\right)$; the reference diameter $\bar{\sigma}$ is taken as the unit of length.

\begin{tabular}{|c|c|c|c|c|}
\hline$m$ & $s$ & $\gamma_{1}$ & $\gamma_{2}$ & $f_{\mathrm{S}}^{\mathrm{ex}}\left(\rho_{\mathrm{S}}^{0}\right)$ \\
\hline \multicolumn{5}{|c|}{$p=0.000, \rho_{\mathrm{S}}^{0}=0.55$} \\
\hline$\ldots$ & $\ldots$ & $\ldots$ & $\ldots$ & 8.289 \\
\hline \multicolumn{5}{|c|}{$p=0.050, \rho_{\mathrm{S}}^{0}=0.55$} \\
\hline 1.00 & 0.0500 & 0.104 & 0.0218 & 8.420 \\
\hline 1.00 & 0.0506 & 0.142 & -0.0278 & 8.457 \\
\hline 1.00 & 0.0497 & 0.155 & -0.0155 & 8.428 \\
\hline 0.999 & 0.0503 & 0.215 & 0.0789 & 8.412 \\
\hline 1.00 & 0.0502 & 0.169 & -0.0637 & 8.417 \\
\hline \multicolumn{5}{|c|}{$p=0.070, \rho_{\mathrm{S}}^{0}=0.57$} \\
\hline 1.00 & 0.0686 & 0.198 & 0.147 & 9.241 \\
\hline 1.00 & 0.0687 & 0.189 & 0.00208 & 9.277 \\
\hline 1.00 & 0.0699 & 0.154 & -0.0471 & 9.248 \\
\hline 1.00 & 0.0705 & 0.294 & 0.207 & 9.234 \\
\hline 1.00 & 0.0692 & 0.186 & 0.0556 & 9.328 \\
\hline \multicolumn{5}{|c|}{$p=0.090, \rho_{\mathrm{S}}^{0}=0.60$} \\
\hline 1.00 & 0.0919 & 0.274 & 0.184 & 10.66 \\
\hline 1.00 & 0.0910 & 0.278 & 0.106 & 10.62 \\
\hline 0.999 & 0.0907 & 0.302 & 0.212 & 10.59 \\
\hline 1.00 & 0.0900 & 0.291 & 0.170 & 10.61 \\
\hline 1.00 & 0.0910 & 0.283 & 0.0910 & 10.70 \\
\hline \multicolumn{5}{|c|}{$p=0.093, \rho_{\mathrm{S}}^{0}=0.65$} \\
\hline 0.997 & 0.0929 & 0.281 & 0.0287 & 12.76 \\
\hline 1.00 & 0.0922 & 0.281 & 0.203 & 13.09 \\
\hline 1.00 & 0.0930 & 0.292 & 0.181 & 13.07 \\
\hline 1.00 & 0.0940 & 0.250 & 0.119 & 13.03 \\
\hline 0.998 & 0.0916 & 0.237 & 0.0179 & 12.76 \\
\hline
\end{tabular}

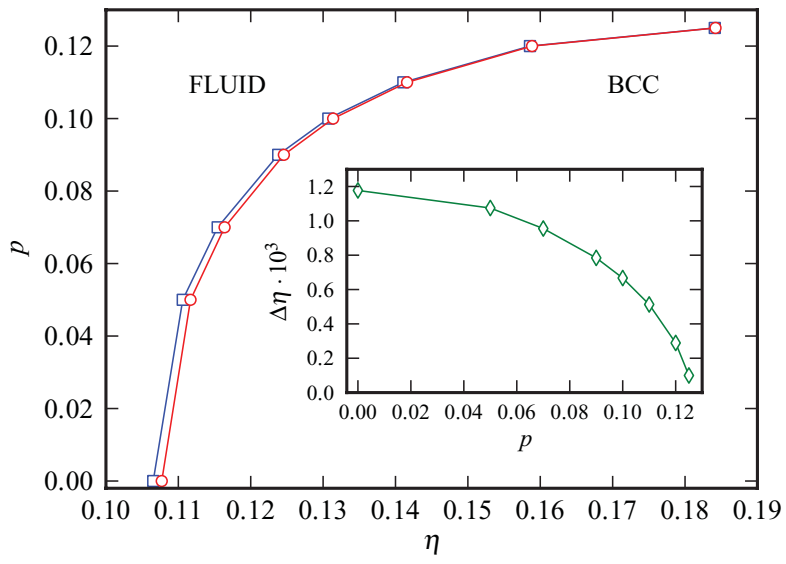

FIG. 3. Quenched-polydispersity phase diagram of hard-core Yukawa particles with $\beta \bar{\epsilon}=81, \kappa \bar{\sigma}=10 / 3$ (system B) presented in the packing fraction $\eta$ - polydispersity $p$ plane. Symbols and labels are the same as in Fig. 1. "BCC" denotes the stable body-centered-cubic crystal phase. Re-entrant melting at a packing fraction $\eta \approx 0.285$ (not reported in the plot for graphical convenience) was found at polydispersity $p=0.125$. Simulations with $p=0.130$ were performed and showed that in that case a fluidlike, disordered state is always stable with respect to the ordered solid. 


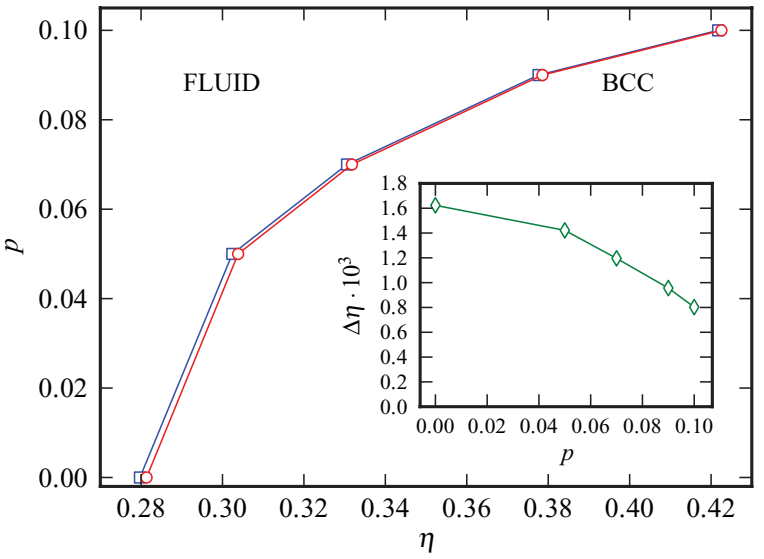

FIG. 4. Quenched-polydispersity phase diagram of hard-core Yukawa particles with $\beta \bar{\epsilon}=20, \kappa \bar{\sigma}=10 / 3$ (system C) presented in the packing fraction $\eta$ - polydispersity $p$ plane. Symbols and labels are the same as in Fig. 1. See the text for remarks on this figure.

Figure 4 reports the phase behavior of model system C with a contact value of the pair potential identical to system A, $\beta \bar{\epsilon}=20$, and inverse screening length identical to model B, i.e., $\kappa \bar{\sigma}=3.333$. Although the shape of the phase diagram is in line with the previous two, the phase boundaries could not be delineated here to their full extent, nor the terminal polydispersity could be located. Indeed, beyond $p=0.1$, the highest value of polydispersity considered, we could not evaluate the free energy of the solid because the procedure outlined in Sec. III B failed to produce an initial crystal configuration with a density $\rho_{\mathrm{s}}^{0}$ high enough for the crystal to be stable against melting.

Figure 5 shows the phase diagram of model system D, characterized by a highly repulsive long-ranged interparticle potential. The general trend uncovered in the previous systems is fully confirmed even in this extreme case. Moreover, our results confirm that an extremely dilute crystal $\left(\eta \approx 10^{-3}\right)$

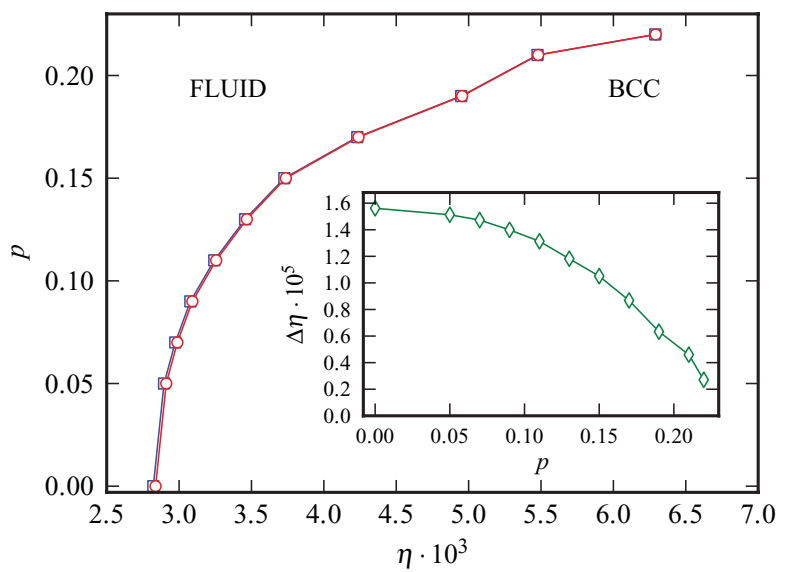

FIG. 5. Quenched-polydispersity phase diagram of hard-core Yukawa particles with $\beta \bar{\epsilon}=1200, \kappa \bar{\sigma}=0.7$ (system D) presented in the packing fraction $\eta$ - polydispersity $p$ plane. Symbols and labels are the same as in Fig. 1. Note the difference in the packing fraction scale between this figure and the previous three. The highest degree of polydispersity investigated for which fluid-solid coexistence was found is $p=0.22$; simulations with $p=0.23$ were performed but in that case a fluidlike, disordered state is always stable over the ordered solid.

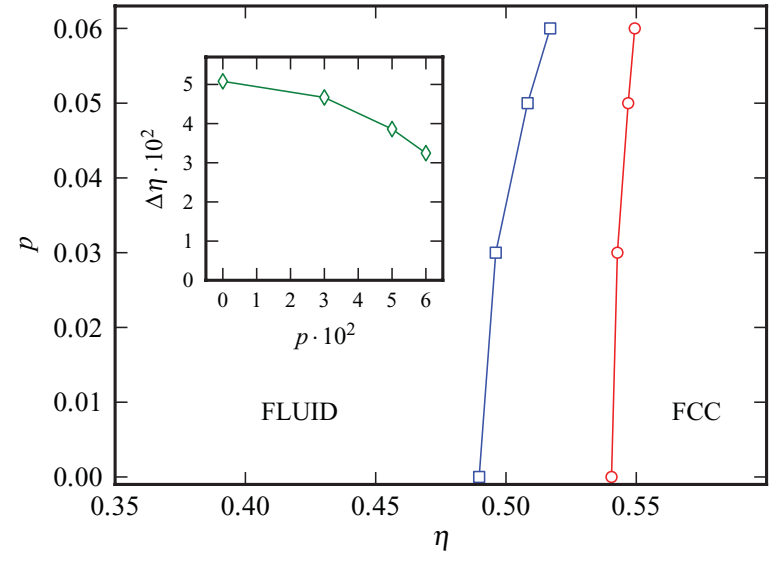

FIG. 6. Quenched-polydispersity phase diagram of polydisperse hard spheres presented in the packing fraction $\eta$ - polydispersity $p$ plane. Symbols and labels are the same as in Fig. 1. Note the difference in the packing fraction scale between this figure and the previous four. The highest degree of polydispersity investigated for which fluid-solid coexistence was found is $p=0.06$.

can sustain a polydispersity as high as $20 \%$ without melting, a fact that substantiates the findings of Leunissen et al. ${ }^{44}$ The terminal polydispersity is indeed quite large and can be bracketed as $0.22<p_{t}<0.23$. In the range of pressures we investigated (rather limited) no evidence was found of re-entrant melting.

For comparison, we also map out the phase diagram for polydisperse hard spheres using the same procedures. The phase diagram is presented in Fig. 6. We again find that the freezing transition shifts toward higher packing fractions and that the difference between the packing fraction of the coexisting solid and fluid phase decreases upon increasing the polydispersity. For polydispersities $p>0.06$, we were not able to construct a crystalline phase using the procedure as described above or by compressing a polydisperse crystal phase to higher densities while retaining the crystalline order (no melting). Hence, we are not able to investigate whether or not a terminal polydispersity exists for hard spheres.

A comparative plot of the effects of polydispersity on the location of the fluid-solid transition and on the width of the coexistence region in the four model systems and in a hard-sphere system is presented in Fig. 7. In the left panel, we plot the relative shift $\left(\bar{\eta}-\bar{\eta}_{0}\right) / \bar{\eta}_{0}$ of the fluid-solid transition as a function of the polydispersity $p$, where $\bar{\eta}=\left(\eta_{\mathrm{F}}+\eta_{\mathrm{S}}\right) / 2$ is the mean packing fraction of the phase separated system. In the right panel, we present the difference $\Delta \eta / \Delta \eta_{0}$ between the packing fraction of the solid and the fluid phase normalized by the corresponding value at $p=0$ as a function of $p$. We find that the normalized difference increases with decreasing $\kappa \bar{\sigma}$.

\section{B. Microscopic structure of the polydisperse solid}

A further question that can be addressed concerns the degree of microscopic order in the polydisperse solid. The solid is initially built as a perfect crystal, with FCC or BCC structure, but in the course of a simulation the particles are free to rearrange themselves. To this end, we consider the four state 

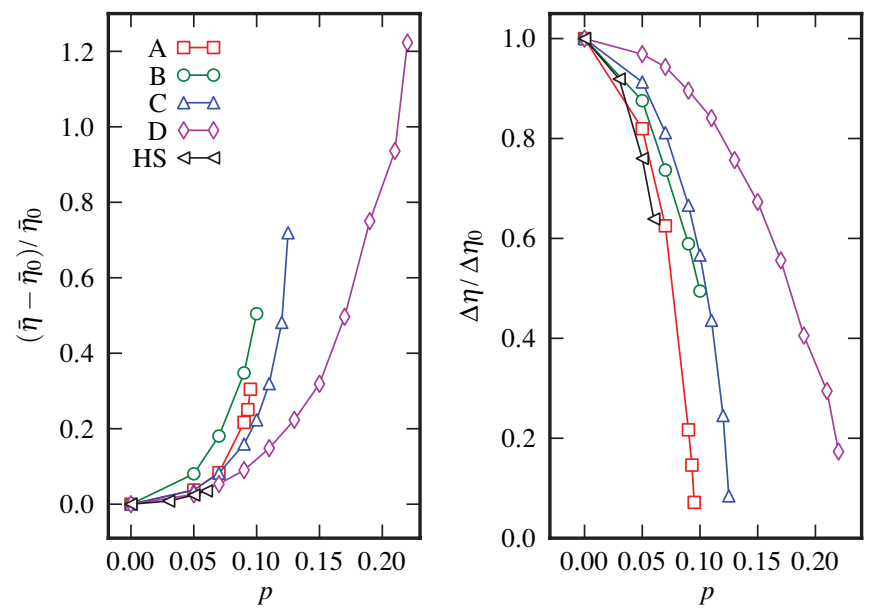

FIG. 7. Comparative plot of the effect of polydispersity in systems A-D and a hard-sphere system. Symbols are the results of Monte Carlo simulations, lines are guides to the eye. (Left panel) Relative shift $\left(\bar{\eta}-\bar{\eta}_{0}\right) / \bar{\eta}_{0}$ of the fluidsolid transition as a function of the polydispersity $p: \bar{\eta}=\left(\eta_{\mathrm{F}}+\eta_{\mathrm{S}}\right) / 2$ is the mean packing fraction of the phase separated system, $\bar{\eta}_{0}$ is the value of the same quantity in the absence of polydispersity. (Right panel) Difference between the packing fraction of the solid and the fluid $(\Delta \eta)$ normalized by the corresponding value at $p=0\left(\Delta \eta_{0}\right)$ plotted as a function of the polydispersity $p$.

points $\mathrm{W}, \mathrm{X}, \mathrm{Y}, \mathrm{Z}$ in the phase diagram of model system A (see Fig. 1) and analyze the microscopic structure of the system at each point. The selected points correspond to a value of polydispersity ( $p=0.093$ ) close to $p_{t}$ : W and $\mathrm{X}$ identify the coexisting fluid and solid phase, respectively, corresponding to the main freezing transition, whereas $\mathrm{Y}$ and $\mathrm{Z}$ correspond to the coexisting solid and fluid phase associated with the reentrant melting transition.

The radial distribution function corresponding to state point $\mathrm{W}$, computed considering all pairs of particles irrespective of their size, is depicted in the upper panel of Fig. 8 and
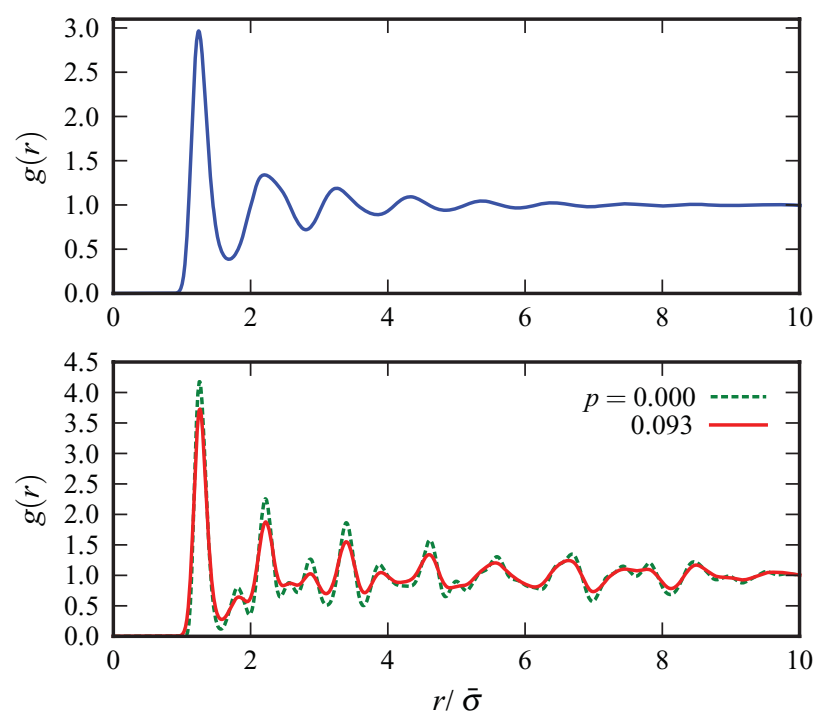

FIG. 8. Upper panel: radial distribution function $g(r)$ as a function of distance $r$ of model A at state point W in Fig. 1. Lower panel: radial distribution function $g(r)$ of model A at state point X (full line) compared to that of a monodisperse system at the same density (dashed line). shows a low degree of coordination as one expects in a fluid state. On the contrary, the radial distribution function of the solid across the phase boundary (state point X), reported in the lower panel, shows a much higher degree of coordination and the features typical of a crystalline arrangement. As a comparison, the radial distribution function of a monodisperse system with the same density is superimposed in the same plot: apart from a certain amount of smearing due to polydispersity in the former case, the two have the same structure. The radial distribution functions corresponding to state points $\mathrm{Y}$ and $\mathrm{Z}$ exhibit the same features and are not shown.

As a further inquiry on the microscopic structure of the system, we perform an analysis of the local degree of crystalline order by employing the $Q_{6}$ bond order parameter. ${ }^{45-47}$ This is one of a series of order parameters that can be used to distinguish fluid and solid domains in a system of particles, exploiting the fact that in a solid the local environments of neighboring particles are strongly correlated, whereas the same correlation is much less pronounced in a fluid sample. More precisely, we associate with each particle a set of vectors-hereafter called bonds-by drawing lines between the particle and each of its neighbors. We then investigate the degree of correlation between the sets of bonds belonging to neighboring particles.

To this end, we project the local particle density around each particle in the sample onto the set of spherical harmonics $\left\{Y_{l}^{m}\right\}$ : although an exact expansion would require harmonics of all orders, we make the hypothesis that the set of harmonics corresponding to a single, suitably chosen order $l$ suffices for our needs. Accordingly, we associate with each particle $i$ a set of complex numbers $\left\{q_{l, m}(i)\right\}$ with $m \in[-l, l]$ :

$$
q_{l, m}(i) \doteq \frac{1}{N_{\mathrm{nb}}(i)} \sum_{j=1}^{N_{\mathrm{nb}}(i)} Y_{l}^{m}\left(\hat{\boldsymbol{r}}_{i j}\right),
$$

where the sum runs over all $N_{\mathrm{nb}}(i)$ neighbors of the particledefined for instance as the particles lying within the first minimum of the radial distribution function-and $\hat{\boldsymbol{r}}_{i j}$ are the orientations of the bonds linking the particle with its neighbors.

The degree of correlation between the environments of two neighboring particles $i$ and $j$ may be quantified by the following normalized scalar product, hereafter called bond correlation index:

$$
S_{i j}=\frac{\sum_{m=-l}^{l} q_{l, m}(i) q_{l, m}^{*}(j)}{\left(\sum_{m=-l}^{l}\left|q_{l, m}(i)\right|^{2}\right)^{1 / 2}\left(\sum_{m=-l}^{l}\left|q_{l, m}(j)\right|^{2}\right)^{1 / 2}}
$$

where $*$ denotes complex conjugation. Note that, owing to the property $q_{l, m}^{*}=(-1)^{m} q_{l,-m}$, the latter is a real quantity.

In the upper panel of Fig. 9 we show the histogram of the bond correlation index $S_{i j}$ in two systems of model A - a monodisperse fluid and a monodisperse FCC crystalfor two different symmetry indices $l$ of the harmonic base $(l \in\{4,6\})$. We notice that in either case the distribution in the fluid is much broader than the one pertaining to the crystal, 

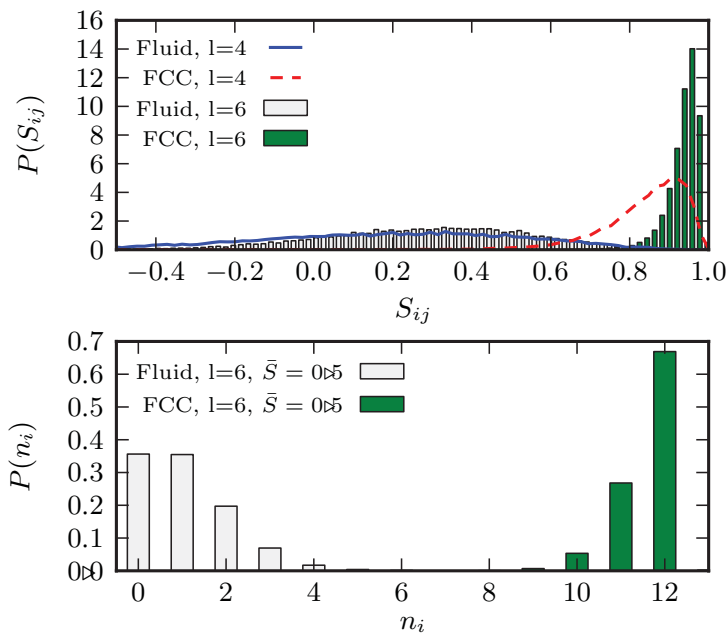

FIG. 9. Distribution of bond order parameters. Upper panel: distribution of the bond correlation index $S_{i j}$ in a monodisperse fluid and in a monodisperse FCC crystal of model A corresponding to $l=4$ and 6 of the (incomplete) harmonic base. Lower panel: distribution of the number of neighbors per particle $n_{i}$ with bond correlation index exceeding the threshold value $\bar{S}=0.5$; the order of the spherical harmonics is chosen as $l=6$.

which is peaked toward the upper end of the horizontal axis; moreover, the distinction is sharper when $l=6$. One may also consider spherical harmonics of higher order: although this is possible, it is not advantageous, since the order parameter may become very sensitive to fluctuations in the position of the particles leading to a broadening of the distribution. Therefore, in the following we will use $l=6$, which enables a clear distinction to be drawn between a fluid and a crystal and, at the same time, is low enough to be robust against fluctuations.

In order to identify the fluid and crystal domains in the system, we pick a threshold value $\bar{S}$ for the bond correlation index and associate with each particle $i$ the number $n_{i}$

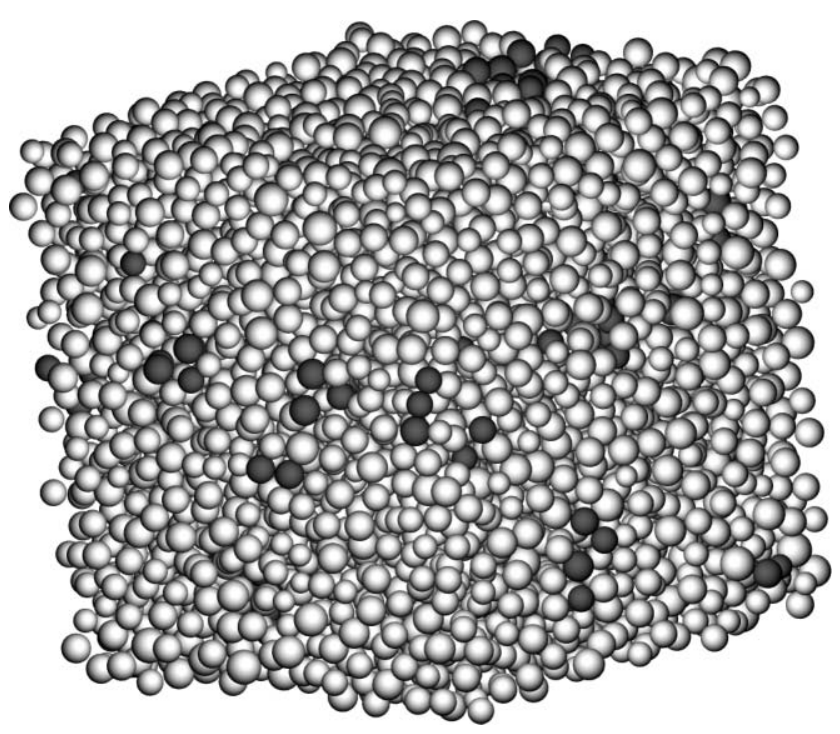

FIG. 10. Snapshot of the system corresponding to the fluid state point W of Model A in Fig. 1; polydispersity is $p=0.093$. Particles are colored according to their classification based on the $Q_{6}$ bond order parameter (see the text for details): light particles are fluidlike, dark particles are solidlike. The crystalline fraction of the system is $3.9 \%$.

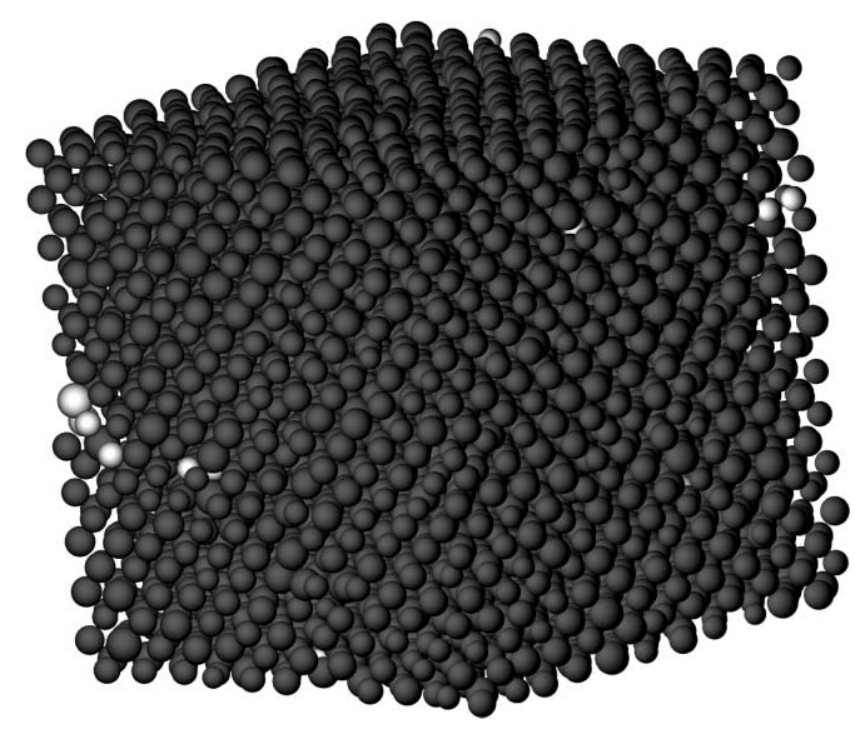

FIG. 11. Snapshot of the system corresponding to crystal state point $\mathrm{X}$ of Model A in Fig. 1; polydispersity is $p=0.093$. Colors have the same meaning as in Fig. 10. The crystalline fraction equals $99.4 \%$.

of neighboring particles for which the bond correlation index exceeds the threshold. In other words, $n_{i}$ is defined as the cardinality of the set $\left\{S_{i j} \mid S_{i j}>\bar{S}\right\}$, where the index $j$ runs over the neighbors of particle $i$; we call this latter quantity the number of correlated neighbors. In the lower panel of Fig. 9 we show the histogram of $n_{i}$ for the two systems already considered, having fixed $l=6$ and $\bar{S}=0.5$. The distributions shown in the panel suggest that we may use the number of correlated neighbors as a practical means to distinguish fluidlike particles from solidlike particles employing a threshold value $\bar{n}$ in the range $[5-8]$; in the following we will take $\bar{n}=6$. $^{59}$

The bond order parameter just introduced allows us to estimate the crystalline fraction of the system, that is the fraction of solidlike particles over the total number of particles, even in the presence of polydispersity: in particular, we apply the procedure to the four state points $\mathrm{W}-\mathrm{Z}$ in the phase diagram of model system A. The two fluid configurations (state points $\mathrm{W}$ and Z) are obviously characterized by a low crystalline fraction, namely $3.9 \%$ and $4.7 \%$. Conversely, the crystalline fraction corresponding to the solid configurations (state points $\mathrm{X}$ and Y) is surprisingly high: $99.4 \%$ and $97.7 \%$, respectively. This shows that the solid retains the full degree of crystalline order even close to the terminal polydispersity. Snapshots of the system with the particles colored according to the bond order parameter are presented for state points $\mathrm{W}$ and $\mathrm{X}$ in Figs. 10 and 11, respectively.

\section{CONCLUSIONS}

We have studied the effect of quenched polydispersity on the freezing transition of repulsive hard-core Yukawa fluids. A theoretical analysis showed that the conditions for polydisperse two-phase equilibrium reduce under the constraint of quenched polydispersity to the equivalent conditions for a monodisperse system. Monte Carlo simulations allowed us to map out the phase diagram of four model systems which span a broad spectrum of interactions, from hard-spherelike to 
highly repulsive. In all systems polydispersity shifts the freezing transition toward higher packing fractions and narrows the density gap between the fluid and the solid. Our results confirm the existence of a terminal polydispersity beyond which the freezing transition disappears, so that a disordered, fluidlike configuration - which eventually may give rise to a glassy state-is stable across the whole density range. Close to the terminal polydispersity the phenomenon of re-entrant melting takes place. Finally, an analysis of the microscopic structure of the system showed that across the freezing transition the polydisperse solid is fully crystalline.

The freezing transition for monodisperse Yukawa systems is well-described by a well-established empirical freezing criterion, ${ }^{48} \Gamma \equiv v(\xi)\left(1+\kappa \xi+\frac{1}{2}(\kappa \xi)^{2}\right) / k_{B} T$ $=106$, with $\xi=(V / N)^{1 / 3}$ the typical particle spacing and $v(r)$ the Yukawa potential between a pair of colloids at centerto-center distance $r$. One might expect to find a similar empirical freezing criterion for polydisperse systems with $v(\xi)$ replaced by either the pair interaction of two reference particles or averaged over the size distributions of the two particles. Unfortunately, we could not find a simple empirical freezing criterion for polydisperse systems.

We stress again that the assumption of quenched polydispersity is fundamental for the previous results to hold. If particle fractionation could take place, the scenario may radically change. Indeed, recent studies employing specialized Monte Carlo simulation techniques ${ }^{49}$ suggest that in this more general case no terminal polydispersity exists, since the system can always freeze by splitting off a solid with a narrow size distribution. ${ }^{50,51}$ It will be interesting to investigate the effect of fractionation on the phase diagram in future work.

\section{ACKNOWLEDGMENTS}

This work was supported by DFG/FOM within SFB TR6 and a NWO-VICI grant. J.C. gratefully acknowledges the Soft Condensed Matter group at the Debye Institute for Nanomaterials Science for the period he spent there, during which most of this work was actually done.

${ }^{1} \mathrm{~J}$. Percus, The Many-Body Problem: Proceedings of the Symposium on the Many-Body Problem, held at Stevens Institute of Technology, Hoboken, New Jersey, January 28-29, 1957 (Interscience, New York, 1963).

${ }^{2}$ B. J. Alder and T. E. Wainwright, J. Chem. Phys. 33, 1439 (1960).

${ }^{3}$ W. W. Wood and J. D. Jacobson, J. Chem. Phys. 27, 1207 (1957).

${ }^{4}$ W. G. Hoover, J. Chem. Phys. 49, 3609 (1968).

${ }^{5}$ P. N. Pusey and W. van Megen, Nature (London) 320, 340 (1986).

${ }^{6} \mathrm{R}$. J. Hunter, Foundations of Colloid Science (Oxford University Press, New York, 2001).

${ }^{7} \mathrm{H}$. Ohshima, Theory of Colloid and Interfacial Electric Phenomena (Academic Press, Amsterdam, 2006).

${ }^{8}$ C. P. Royall, M. E. Leunissen, A.-P. Hynninen, M. Dijkstra, and A. van Blaaderen, J. Chem. Phys. 124, 244706 (2006).

${ }^{9}$ D. Hone, S. Alexander, P. M. Chaikin, and P. Pincus, J. Chem. Phys. 79, 1474 (1983)

${ }^{10}$ C. F. Tejero, J. F. Lutsko, J. L. Colot, and M. Baus, Phys. Rev. A 46, 3373 (1992).

${ }^{11}$ A.-P. Hynninen and M. Dijkstra, Phys. Rev. E 68, 021407 (2003).

${ }^{12}$ P. Sollich, J. Phys. Condens. Matter 14, R79 (2002).

${ }^{13}$ H. J. Schöpe, G. Bryant, and W. van Megen, J. Chem. Phys. 127, 084505 (2007).

${ }^{14}$ E. Zaccarelli, C. Valeriani, E. Sanz, W. C.K. Poon, M. E. Cates, and P. N. Pusey, Phys. Rev. Lett. 103, 135704 (2009).

${ }^{15}$ M. Hermes and M. Dijkstra, Europhys. Lett. 89, 38005 (2010).
${ }^{16}$ T. Nogawa, N. Ito, and H. Watanabe, Phys. Rev. E 82, 021201 (2010).

${ }^{17}$ N. B. Simeonova and W. K. Kegel, Phys. Rev. Lett. 93, 035701 (2004).

${ }^{18}$ M. E. Leunissen, M. T. Sullivan, P. M. Chaikin, and A. van Blaaderen, J. Chem. Phys. 128, 164508 (2008).

${ }^{19}$ P. N. Pusey and W. van Megen, Phys. Rev. Lett. 59, 2083 (1987).

${ }^{20}$ S. R. Williams, I. K. Snook, and W. van Megen, Phys. Rev. E 64, 021506 (2001).

${ }^{21}$ T. M. Truskett, S. Torquato, and P. G. Debenedetti, Phys. Rev. E 62, 993 (2000).

${ }^{22}$ S. I. Henderson, T. C. Mortensen, S. M. Underwood, and W. van Megen, Physica A 233, 102 (1996).

${ }^{23}$ R. McRae and A. D. J. Haymet, J. Chem. Phys. 88, 1114 (1988).

${ }^{24}$ J.-L. Barrat and J.-P. Hansen, J. Phys. (Paris) 47, 1547 (1986).

${ }^{25}$ P. Bartlett and P. B. Warren, Phys. Rev. Lett. 82, 1979 (1999).

${ }^{26}$ P. Bartlett, J. Chem. Phys. 107, 188 (1997).

${ }^{27}$ P. Bartlett, J. Chem. Phys. 109, 10970 (1998).

${ }^{28}$ R. P. Sear, Europhys. Lett. 44, 531 (1998).

${ }^{29}$ M. Fasolo and P. Sollich, Phys. Rev. Lett. 91, 068301 (2003).

${ }^{30}$ P. N. Pusey, E. Zaccarelli, C. Valeriani, E. Sanz, W. C. K. Poon, and M. E. Cates, Philos. Trans. R. Soc. London 367, 4993 (2009).

${ }^{31}$ N. B. Wilding, J. Chem. Phys. 130, 104103 (2009).

${ }^{32}$ N. B. Wilding, P. Sollich, M. Fasolo, and M. Buzzacchi, J. Chem. Phys. 125, 014908 (2006)

${ }^{33}$ S. Phan, W. B. Russel, J. Zhu, and P. M. Chaikin, J. Chem. Phys. 108, 9789 (1998).

${ }^{34}$ P. G. Bolhuis and D. A. Kofke, Phys. Rev. E 54, 634 (1996).

${ }^{35}$ D. A. Kofke and P. G. Bolhuis, Phys. Rev. E 59, 618 (1999).

${ }^{36}$ J. J. Salacuse, J. Chem. Phys. 77, 3714 (1982).

${ }^{37} \mathrm{H}$. B. Callen, Thermodynamics and an Introduction to Thermostatistics (Wiley, New York, 1985).

${ }^{38}$ J. J. Salacuse, J. Chem. Phys. 81, 2468 (1984).

${ }^{39}$ D. Frenkel and B. Smit, Understanding Molecular Simulation, Second Edition: From Algorithms to Applications, Computational Science Series Vol 1 (Academic Press, Amsterdam, 2001).

${ }^{40}$ D. Frenkel and A. J. C. Ladd, J. Chem. Phys. 81, 3188 (1984).

${ }^{41}$ J. M. Polson, E. Trizac, S. Pronk, and D. Frenkel, J. Chem. Phys. 112, 5339 (2000).

${ }^{42}$ T. N. L. Patterson, Math. Comput. 22, 847 (1968).

${ }^{43}$ R. Piessens, Quadpack: A Subroutine Package for Automatic Integration (Springer-Verlag, Berlin, 1983).

${ }^{44}$ M. E. Leunissen, A. van Blaaderen, A. D. Hollingsworth, M. T. Sullivan, and P. M. Chaikin, Proc. Natl. Acad. Sci. 104, 2585 (2007).

${ }^{45}$ P. J. Steinhardt, D. R. Nelson, and M. Ronchetti, Phys. Rev. B 28, 784 (1983).

${ }^{46}$ S. Auer and D. Frenkel, Adv. Comput. Simul. 173, 149 (2005).

${ }^{47}$ W. Lechner and C. Dellago, J. Chem. Phys. 129, 114707 (2008).

${ }^{48}$ O. S. Vaulina and S. A. Khrapak, J. Exp. Theor. Phys. 90, 287 (2000).

${ }^{49}$ N. B. Wilding and P. Sollich, J. Chem. Phys. 133, 224102 (2010).

${ }^{50}$ M. Fasolo and P. Sollich, Phys. Rev. E 70, 041410 (2004).

${ }^{51}$ P. Sollich and N. B. Wilding, Phys. Rev. Lett. 104, 118302 (2010).

${ }^{52}$ For an interesting discussion on the connection between random and polydisperse systems see Ref. 38.

${ }^{53}$ The constraint prevents a global translational motion of the crystal, so that the particles do not drift away from the crystal lattice. To implement the constraint in a polydisperse system no information on the mass of the particles is required, since a common effective mass $\bar{m}$, which eventually cancels out, can be assigned to each particle.

${ }^{54}$ More precisely: to compute the equations of state we perform simulations at fixed pressure and average the resulting volumes. In the case of the Einstein integration we average the excess solid free energies.

${ }^{55}$ Typically, we set $r_{c}$ so that the pair potential is less than $10^{-5} k_{B} T$ at the cutoff.

${ }^{56}$ If the cores do not feel each other, $N^{-1}\left\langle\beta U_{\lambda}\right\rangle_{\lambda}^{\mathrm{CM}}$ should approach $3 / 2$ as $\lambda \rightarrow 1$.

${ }^{57}$ We were only able to determine a reentrant behaviour for $p=0.093$ and $p=0.095$; the curve corresponding to $p=0.090$ in Fig. 2 might cross the zero axis a second time if extended to high enough pressures. In any case, we expect the phenomenon of reentrant melting to be limited to a narrow interval below $p_{t}$.

${ }^{58}$ Since no simulation was performed for $p=0.100$, a safer upper limit, as deduced from Fig. 2, is $p_{t}<0.105$.

${ }^{59}$ In other words, we consider a particle $i$ solid-like if $n_{i}>\bar{n}$, otherwise it is classified as fluid-like. 